Voix et Images

volxetimages

\title{
Les voyages visionnaires de trois créatrices \\ féministes-matristiques : Emily Carr, Jovette Marchessault et Gloria Orenstein
}

\section{Gloria F. Orenstein}

Volume 16, numéro 2 (47), hiver 1991

Jovette Marchessault

URI : https://id.erudit.org/iderudit/200898ar

DOI : https://doi.org/10.7202/200898ar

Aller au sommaire du numéro

\section{Éditeur(s)}

Université du Québec à Montréal

\section{ISSN}

0318-9201 (imprimé)

1705-933X (numérique)

Découvrir la revue

Citer cet article

Orenstein, G. F. (1991). Les voyages visionnaires de trois créatrices

féministes-matristiques : Emily Carr, Jovette Marchessault et Gloria Orenstein.

Voix et Images, 16(2), 253-261. https://doi.org/10.7202/200898ar d'utilisation que vous pouvez consulter en ligne. 


\title{
Les voyages visionnaires de trois créatrices féministes-matristiques: Emily Carr, Jovette Marchessault et Gloria Orenstein *
}

\author{
par Gloria F. Orenstein, University of Southern \\ California
}

Quand j'ai découvert les écrits de Jovette Marchessault, au printemps 1978, je voulus sur le champ écrire quelque chose sur son premier roman, Comme une enfant de la terre et, à cette époque, j'ai intitulé mon article: «Jovette Marchessault ou la quête extatique de la nouvelle chamane féministe" 1 . Pendant les années 70 et 80 , je m'étais servi du mot "chamane" au sens métaphorique et lorsque j'écrivais sur l'artiste en tant que chamane je voulais dire en fait, "artiste visionnaire". À ce moment-là, toutes les connaissances que je possédais du mot «chamane" étaient livresques. Beaucoup de choses ont changé depuis lors et c'est à partir de ces changements que je voudrais commenter la dernière pièce de Jovette Marchessault, le Voyage magnifique d'Emily Carr. Le titre du présent article renvoie donc à l'article originel, rédigé il y a douze ans, et mon intention est d'en élargir le propos.

$\mathrm{Au}$ cours de la dernière décennie, Jovette a écrit de nombreuses pièces, divers romans, essais et articles. Ses sculptures et ses tableaux ont été présentés lors d'expositions individuelles et collectives. L'ensemble de ses créations témoigne d'un talent artistique de plus en plus impressionnant. D'une façon ou d'une autre, l'évolution créative de Jovette ainsi que mon propre développement universitaire et personnel se sont réalisés en tandem ou en parallèle. C'est ainsi que je peux encore une fois parler de la production artistique de Jovette comme étant " chamanique "; mais cette fois je le fais à partir d'une perspective nouvelle, inédite, celle d'une adepte du chamanisme, en même temps que d'une perspective plutôt traditionnelle, celle de la critique littéraire. En fait,

* Cet article a été traduit de l'américain par Donald Bruce.

1 Bulletin de la Société des professeurs de français d'Amérique, I, automne 1979, p. 37-57. 
depuis la nouvelle orientation qu'a prise ma vie, je m'étonne de voir à quel point j'avais raison, il y a douze ans, de parler de la création artistique de Jovette comme étant * chamanique *. Avant d'entamer la discussion de sa pièce, je passerai brièvement en revue les années les plus récentes de notre association littéraire/critique.

À la fin des années 1970 , en tant que professeure féministe de littérature et cofondatrice du Salon des femmes pour la littérature (The Women's Salon for Literature) à New York (1975-1985), je me suis engagée à transformer le rapport entre les spécialistes de la littérature et la nouvelle génération d'écrivaines: d'un rapport partant du soi-disant *jugement objectif * voire de critique négative, à un rapport positif de soutien, d'encouragement et de reconnaissance féministes. Mon intention était de promouvoir la création au lieu de décourager l'exploration des nouveaux domaines dans lesquels les écrivaines féministes s'engageaient.

Une de mes directrices de thèse, Erika Ostrovsky, de l'Université de New York (NYU), m'a fait connaître le travail de Jovette Marchessault. Madame Ostrovsky savait que j'étudiais la mythologie et les arts et que je travaillais sur la réémergence de l'image de la Déesse dans l'art des femmes contemporaines. Pour cette raison, elle pensait que je trouverais intéressantes les sculptures des Femmes Telluriques de Jovette. Ce n'est qu'en passant qu'elle m'a mentionné que Jovette était aussi écrivaine. Après avoir donné des conférences sur les Femmes Telluriques et les Femmes de l'Espoir et de la Résurrection, au colloque de Santa Cruz (printemps 1978) sur le thème "The Great Goddess Re-Emerging ", j’ai demandé à Jovette, plus ou moins au hasard, de me parler de son travail d'écrivaine.

Une semaine plus tard, je recevais une copie de son premier roman, Comme une enfant de la terre, qui avait mérité le prix France-Québec. J'ai tout lu d'une traite et j'ai su tout de suite que j'avais découvert le travail d'une écrivaine très importante. Je me souviens d'une lettre que j'ai écrite à Jovette, à l'époque, dans laquelle je lui disais :

Je ne sais pas si ce que je vous dis vous intéresse, car je ne suis qu'une jeune professeure adjointe, totalement inconnue. Pourtant, je voudrais vous dire que je reconnais dans votre roman un grand texte littéraire, un des meilleurs livres que j'aie lus dans ma vie. Qui êtes-vous? Qu'avez-vous écrit d'autre? Il me faut absolument écrire sur vous.

Quelques jours plus tard, le manuscrit de la Mère des herbes arrivait chez moi. Encore un livre brillant. Bien que j'aie été à la veille de mon départ pour la France où j'allais diriger le programme de l'université Rutgers pour les étudiants de troisième année et qu'il ne me restât qu'un mois, je m'étais résolue à écrire un article sur les 
livres de Jovette. Mais que voulait dire le fait que la protagoniste de son premier roman traversait le continent amérindien dans le ventre d'un Greyhound accompagné de Thunderbird, Little Crow et Cloud Head? Je savais qu'il faudrait percer ce mystère, mais à l'époque je ne savais rien du chamanisme.

Un jour je suis entrée dans mon bureau (où logeait alors une jeune étudiante en beaux-arts qui se croyait d'origine franco-canadienne/ amérindienne), quand un livre est tombé d'une étagère sur ma table de travail. Puisque bon nombre de mes livres avaient été recouverts par des photos, les œuvres d'art et les objets amérindiens et francocanadiens de l'étudiante, je ne me souvenais plus des livres reposant sur les étagères. Celui qui m'est ainsi tombé dans les mains le jour où j'ai commencé à écrire sur le travail de Jovette était The Ghost Dance Religion (la Religion de la danse des revenants) de Weston La Barre. Je ne savais même pas que je possédais un tel livre! Je ne savais pas non plus de quoi il s'agissait. En lisant le livre, j'ai découvert le voyage visionnaire d'une chamane amérindienne. D'un coup je me suis rendu compte qu'il existait un parallèle entre le voyage visionnaire de la chamane et celui de l'écrivaine. Peu après, j'écrivais que la nouvelle chamane féministe voyagerait dans un " autre monde", dans une autre dimension de la réalité, accompagnée de ses guidesesprits, qu'elle prendrait contact avec les habitants du monde des esprits, les déesses, les foremothers, et les modèles de sagesse, et qu'elle reviendrait dans notre dimension, éclairée et munie de nouvelles puissances, porteuse d'une nouvelle vision transformatrice pour sa communauté. Ensuite, la nouvelle chamane féministe voyagerait à la fois de manière physique (dans le Greyhound) et de manière spirituelle (dans le ventre d'un lévrier) avec ses "animaux de puissance» (Thunderbird, Little Crow, Cloud Head), et retrouverait notre Déesse perdue, la Sagesse. Quelques années plus tard, Jovette écrivait Vaches de nuit, qui devait être mis en scène par Pol Pelletier au Salon des femmes à New York. Cependant, au fur et à mesure que sa création chamanique remplissait l'espace de ma vie et celui de mon salon féministe, il ne m'est pas venu une seule fois à l'esprit que ce que j'avais écrit sur le mot " chamane " préfigurerait des événements de ma propre vie - et que ce serait moi qui serais appelée, littéralement et non de façon métaphorique, à suivre le chemin de la chamane.

Ce n'est qu'en janvier 1987, au moment où Ellen Maret GaupDunfjeld, chamane du Samiland (la partie norvégienne de la Laponie), s'est présentée dans mon bureau à l'Université de Santa Cruz, que j'ai appris qu'on m'appelait à mon tour à entreprendre mon propre voyage chamanique-féministe. Donc, depuis trois ans et demi, j'étudie le chamanisme Sami (lapon) et pendant cette période j'ai fait quatre voyages au pôle Nord; ma professeure chamane m'a rendu visite autant de fois aux États-Unis. 
Le présent article part de ces nouvelles connaissances du chamanisme, élargies et fondées sur l'expérience directe que j'en ai acquise grâce à l'apprentissage que j'en fais à présent.

Afin d'expliquer en quoi ou pourquoi je crois que la création artistique de Jovette Marchessault differe d'autres œuvres artistiques contemporaines, je dois raconter une dernière anecdote. Une artiste de la Californie, Jean Edelstein, travaille avec une chamane coréenne qui est également danseuse. En public, Jean Edelstein pratique le tachisme (Action Painting) selon les rythmes des danses de la chamane. Elle avait auparavant fait du tachisme avec d'autres danseuses, mais jamais avec une chamane. Depuis qu'elle travaille avec la chamane, elle commence à franchir certaines limites, ce qui remet en cause la définition occidentale de l'art. Par exemple, au début de cette relation, Jean effaçait ou redonnait parfois un coup de pinceau. La chamane s'est sentie blessée et a interdit à Jean de recommencer. La réponse de Jean fut alors que c'était elle l'artiste, et que l'artiste savait bien ce qui était important pour l'œuvre; que la chamane était bien son modèle, mais pas l'artiste, et qu'elle n'avait pas le droit de prendre ces décisions. À la longue, on s'est aperçu que c'était la chamane qui était la professeure de Jean et que, pendant les sessions de travail, celle-ci entrait en une légère transe. Son esprit se confondait, d'une certaine façon, avec celui de la chamane pendant qu'elles peignaient et dansaient ensemble. Jean a également appris que son art avait en quelque sorte vraiment pénétré l'esprit de la chamane, et que le fait d'effacer ou de donner un coup de pinceau altérait réellement son champ d'énergie. La production artistique de Jean est ainsi devenue tout à fait différente: avant de travailler avec une chamane, Jean était «l'artiste * et * son modèle *, une danseuse, indépendante d'elle. Le résultat artistique ainsi obtenu est ce que j'appellerais la "représentation" des mouvements du modèle ou de la danseuse. Ce qui a changé dans le travail de Jean avec la chamane, c'est qu'il n'y a plus de séparation ni de frontière entre ce que nous appelons l'«artiste" et le "modèle". Au contraire, les deux se mêlent, et leurs esprits entrelacés voyagent ensemble pendant le moment extatique de collaboration. Nous pourrions dire aussi que l'esprit de Jean fait des voyages avec sa chamane, et qu'il en rapporte une vision (une œuvre d'art) qui est la trace ou la piste de son propre voyage spirituel au lieu d'être une représentation de la danse du modèle.

Ainsi, l'art de la chamane renverse la conception occidentale de la création qui postule la séparation du créateur et de l'objet représenté, de l'esprit et de la matière: il ne s'agit pas d'une "artiste" qui représente un "objet». Il s'agit plutôt d'un cheminement spirituel où "l'artiste comme chamane " et la "chamane comme artiste" voyagent ensemble dans un état de transe extatique qu'elles partagent. L'œuvre d'art devient alors la carte vivante du voyage, un chemin 
plein d'énergie qui porte la marque du passage des esprits. J'appellerais ce type d'art " totémique " ou "chamanique ". Il ne peut être réduit à une série d'innovations artistiques. Cet art repose sur le fait d'entendre et de voir les dimensions invisibles du monde des esprits qui nous accompagnent dans nos voyages créateurs, dans la vie et dans l'art. L'œuvre est vivante; imbue d'esprit, et, comme les totems dans les tableaux d'Emily Carr et les pièces de Jovette Marchessault, l'œuvre d'art est perçue comme un médium vivant. C'est peut-être pour cette raison que Jovette a choisi le théâtre comme moyen d'expression "par excellence " pour la plupart de ses textes, y compris pour les textes qui n'ont pas été écrits spécifiquement pour la scène, mais qui ont été quand même montés comme Vaches de nuit.

L'expression "féministe-matristique" caractérise la vision cosmique de Jovette Marchessault. Dans sa pièce, c'est la Grande Déesse (D'Sonoqua) qui règne sur l'univers d'Emily Carr, et cette Déesse s'associe à la couleur verte, ce qui symbolise la Mère de la Terre, la Déesse de la végétation. J'aimerais également ajouter le qualificatif "éco-féministe" à ma description de l'œuvre de Jovette. La Maison de toutes les espèces, maison d'Emily, est devenue une sorte de temple qui honore toutes les créatures qui vivent sur la planète et qui sont sacrées aux yeux de la Déesse. Emily est devenue la gardienne et la protectrice de la création de la Mère de la terre. Elle est comme une travailleuse sociale du sacré; quelqu'un qui écoute soigneusement les confessions des animaux, et qui les emmène à la mer ou dans des voyages vers d'autres réalités dont ils ont grand besoin afin de fortifier et de nourrir leur âme.

Comme écrivaine éco-féministe, Jovette Marchessault n'établit jamais de hiérarchie qui séparerait les êtres humains des autres espèces animales ou qui les placerait au-dessus de la nature non humaine, ou encore qui les en séparerait. Au contraire, un univers de Déesse-mère (matristique), éco-féministe, est un univers qui n'est ni androcentrique ni anthropomorphique - un univers où toutes les espèces vivent ensemble en harmonie dans un monde où tout se définit à partir d'images féminines.

La Maison de toutes les espèces est pénétrée de l'esprit de la maternité: celui de la vache de notre enfance, à mes sœurs et à moi. Une grande vache blanche et rousse aux cornes pointues (p. 25) ${ }^{2}$. Sous le regard spirituel de l'esprit d'une Déesse Vache Mère, la Maison de toutes les espèces élève l'esprit du texte Vaches de nuit à une nouvelle dimension. Cette maison est l'expression illimitée de tout ce qui existe sur cette planète (p. 25). Il n'existe plus de séparation entre les

2 Les citations sont tirées du manuscrit prêté par l'auteure. 
"Vaches de nuit" et les "Vaches du jour *. La Déesse Vache Mère contient à la fois la nuit et le jour, puisque tout ce qui vit sur la terre se trouve sous la protection sacrée d'une créatrice; la vie diurne n'est plus vécue comme émasculation, mais comme entretien et habilitation.

Tandis que les visions féministe/matristique et éco-féministe sont des extensions et des développements des premiers textes de Jovette (les Vaches de nuit, Comme une enfant de la Terre, la Saga des poules mouillées, la Mère des herbes, etc.), l'évolution de la perspective chamanique dans cette pièce est unique, car elle révise complètement à la fois notre compréhension de l'art et de la mort. Elle montre clairement comment la création artistique devient la matrice sacrée d'où le voyage de la chamane au monde des esprits émane et comment le voyage de la chamane est la source de nouvelles visions artistiques.

L'univers d'Emily Carr, comme celui de la chamane, est peuplé de guides-esprits tel l'Accordeur d'âmes, et des incarnations de l'esprit de la Grande Déesse de la Terre telle sa chère amie Sophie. L'Accordeur d'âmes, qui sort d'un fourneau (alchimique), apporte littéralement la lumière et la transformation dans la vie d'Emily. Il lui dit qu'il existe au seuil de la vie et de la mort et qu'il n'y a pas de mort. Il lui confirme qu'il a lutté à ses côtés, qu'il lui a présenté ses alliés (le peintre Harris, membre du Groupe des Sept, et D'Sonoqua, la Déesse), et qu'il l'a guidée vers des rencontres avec ses âmes-compagnes, Sophie, Harris et D'Sonoqua.

On peut faire appel à son guide-esprit, l'Accordeur d'âmes, quand on a besoin de lui, et il se présente lors de tous les événements qui impliquent la traversée du seuil du monde des esprits. Les esprits des ancêtres sont également présents dans cette pièce. Ils sont représentés de façon métaphorique par les câbles et les poulies qui montent et font descendre la chaise et la table de son père et de sa mère, ce qui montre le lien toujours présent entre Emily et ses parents décédés. Grâce aux pouvoirs uniques de Sophie, qui sont préservés par les peuples indigènes (qui vivent en accord avec les esprits du monde naturel, et en contact avec le monde des esprits), Emily est capable de communiquer avec l'esprit de sa sœur Lizzie après sa mort.

Dans le premier tableau de la version précédente et non révisée de la pièce (qui est devenu à présent le deuxième tableau), la sœur de Sophie, qui porte le nom d'Emily, vient de mourir. Sophie a besoin d'une pierre tombale pour l'enfant et l'Accordeur d'âmes lui offre une pierre de cristal. Cette pierre tombale faite de cristal symbolise la communication spirituelle directe avec les morts et, comme une radio à cristal, elle sert à transmettre les messages du monde des esprits. Il se peut que la fille de Sophie communique avec celle qui porte le 
même nom, l'artiste Emily Carr, et qu'elle lui transmette les visions et les sons du monde des esprits qu'elle illustre dans son art totémique. Parfois il m'arrive d'entendre la voix des totems: C'est en toi que nous continuons notre existence, disent-ils (p. 17). Lorsque Harris décrit les tableaux d'Emily, il met laccent sur leur qualité phosphorescente et le fait que les vibrations de l'esprit et de la force de la vie les remplissent. Emily dit aussi qu'elle perçoit la vibration éclatante $d u$ vivant (p. 47). Cette perception de l'esprit dans la matière, cette vision animiste, constitue la vision de la chamane. Dans l'art d'Emily Carr, tout comme dans l'univers créateur de Jovette Marchessault, toutes les dimensions, spirituelle, matérielle, humaine et non humaine, se trouvent impliquées dans un grand réseau. L'art, comme une pierre tombale de cristal, reçoit et transmet des messages du monde des esprits. Un tableau, comme un cristal, est réellement une entité vivante. Ce sont des formes de la mémoire.

Le voyage artistique est synonyme du voyage chamanique et le voyage chamanique devient la grande matrice de la vision artistique. Quand Emily rencontre la Déesse, D'Sonoqua, elle voit un éléphant qui est à la fois une caravane et une Déesse. La montagne, elle aussi, fait parvenir ses messages. Une vision chamanique permet à l'individu de voir les formes changeantes et les épiphanies du sacré dans notre monde. Les chamanes voient souvent les animaux de pouvoir et reconnaissent les messages du monde des esprits dans les oiseaux. D'Sonoqua peut se présenter à Emily sous des épiphanies nombreuses - comme éléphant, comme tente-roulotte motorisée ou comme montagne. Il n'y a que les chamanes qui savent que les caravanes, les éléphants, les montagnes sont en effet des épiphanies de la Grande Déesse. Et voir la Déesse dans un éléphant ou dans une caravane est le travail visionnaire de l'artiste, ainsi que celui de la chamane. Un des moments dramatiques de la révélation chamanique est celui où le décor est transformé: D'Sonoqua porte l'auréole et la couleur verte de la Terre-Mère devient manifeste. La théophanie d'Emily la conduit à peindre l'esprit des totems, et les êtres-esprits qui ont ensemencé la terre en lumière.

Dans la vision du monde chamanique, la mort n'est qu'un seuil, un passage dans le monde des esprits. Nous sommes toujours entourés des esprits de nos ancêtres (ce que les chaises et les tables des parents d'Emily attachées à la scène par des poulies et des câbles rendent dans la pièce), et leur présence ainsi que leur intervention spirituelle continuelle dans la vie est presque palpable. Puisque nous sommes rattachés à nos ancêtres, et qu'il n'existe pas de mort mais seulement une transition et une transformation, lorsque Lizzie traverse le seuil du monde des esprits, Sophie peut facilement canaliser les pensées de Lizzie vers Emily et celles d'Emily vers Lizzie. C'est dans la lumière de la forêt qu'Emily a éprouvé la 
sensation d'être accompagnée par les pas de Lizzie. La nature et l'art apparaissent comme des portes qui s'ouvrent sur le monde des esprits. Sophie renforce la conviction d'Emily qu'il n'y a pas de séparation au moment de la mort: Entre une goutte d'eau et sa voisine, $y$-a-t- il séparation? Jamais séparation nulle part. (p. 84)

Ainsi, comme nous l'avons démontré, la création de Jovette Marchessault repense le sens de l'art et de la mort à partir de la perspective chamanique. L'art se comprend maintenant en termes totémiques, il est infusé de messages et de sens spirituels, il est vivant. La pièce de Jovette Marchessault ne traite pas d'Emily Carr. La pièce est l'empreinte du seuil vivant où l'esprit de Jovette s'unit avec celui d'Emily Carr, où les vivants et les morts ont communié. Et dans les tableaux d'Emily Carr, il n'est pas question des esprits de la forêt. Ses toiles sont des totems qui renvoient plutôt à des lieux où les esprits de la forêt lui parlaient et parlaient à travers elle. Afin de pousser l'analogie un peu plus loin, je voudrais ajouter que mes écrits sur l'art de Jovette Marchessault indiquent également ce point de fusionnement, ce moment où mon esprit a voyagé avec le sien aux royaumes des Vaches de nuit, des Poules mouillées, des femmes du Salon et de la grande Déesse. C'est parce que ces œuvres sont vivantes qu'on peut, littéralement, utiliser le mot " création " pour s'y référer.

Le Voyage magnifique d'Emily Carr est plus que le récit d'une vie, car celle-ci dépasse les barrières du temps et de l'espace et continue au-delà de la mort physique. C'est une vie présentée à travers le cadre de l'éternel. Le voyage de l'artiste est un voyage d'initiation vers la connaissance toujours croissante des dimensions illimitées de l'invisible. Cette pièce montre clairement que, tandis que l'art est produit par des êtres humains vivant sur la surface de la terre, sa source est spirituelle. Les archivistes de l'au-delà conservent le dossier des événements et le souvenir des morts. Quand l'Accordeur d'âmes souffle de la poussière météorique sur Emily, elle aussi devient un des scribes célestes inspirés par son guide-esprit, ainsi qu'une des écrivaines qui canalisent les messages du monde des esprits. La vie créatrice et spirituelle d'Emily ne s'achève pas avec sa mort physique. En effet, sa mort devient une renaissance, la plus grande initiation aux mystères de la création.

Finalement, le théâtre comme moyen d'expression peut se comprendre à la fois littéralement et métaphoriquement. Dans le théâtre de l'Antiquité grecque, les acteurs portaient des masques et ils agissaient comme des médiums parce qu'ils devenaient véritablement les dieux ou qu'ils canalisaient la parole des dieux pendant le spectacle. Les masques figeaient une expression de l'éternel sur leur visage. C'est cet éternel visage de la création qu'Emily Carr restaure dans ses tableaux et que Jovette Marchessault redonne au théâtre. De nouveau, l'actrice qui joue le rôle d'Emily Carr sera perçue publi- 
quement comme une médium. Maintenant c'est le texte qui deviendra le masque et qui créera l'image de l'éternel dans la vie de ceux et celles qui assistent au spectacle. En effet, ce théâtre nous initie à la connaissance de la vie qui existe au-delà des confins terrestres du temps et de l'espace. La pièce de Jovette Marchessault fonctionne comme le masque dans le théâtre grec, masque qui permet à l'esprit de parler à travers elle. Cependant, ici, le masque est réel. Bien entendu, c'est la perception limitée de la vie humaine, coupée de la dimension spirituelle, qui en constitue la déception. L'art chamanique démasque cette déception et renvoie l'image de la vie éternelle à la scène pour que nous puíssions nous contempler à nouveau dans le contexte cosmique de notre existence.

Le voyage visionnaire, que je fais depuis douze ans à l'aide de la production créatrice de Jovette Marchessault qui me sert de guide spirituel, m'a rendu le pouvoir qui accompagne la reconnaissance du fait que, l'imaginaire est ce qui tend à devenir réel comme l'ont dit les surréalistes. En fait, le voyage imaginaire est devenu de plus en plus réel et sa dimension chamanique est portée par des éléments aussi variés que l'éléphant, la caravane, le fourneau, le livre, le tableau, la pièce ou le texte critique. C'est ainsi que la critique chamanique voyage aussi au moyen des arts au royaume sacré de la Déesse et rentre imbu d'une vision pour sa communauté. J'espère que les lecteurs et lectrices comprendront maintenant que ce texte-ci, comme la pièce de Jovette et les tableaux d'Emily Carr, trace le chemin d'un voyage spirituel et qu'il est vivant. Comme un fourneau alchimique, qui est également une locomotive, il devra devenir un moyen de transport grâce auquel les lecteurs et les lectrices voyageront à travers les œuvres de Jovette Marchessault et d'Emily Carr, de la réalité manifeste aux dimensions de l'invisible, chargé de la compréhension que tout est à la fois réel et sacré, que la création est illimitée et que la Déesse refleurit. 